\title{
Análise da Revisão Cochrane: Fixação com Parafusos Pediculares de Fraturas Traumáticas da Coluna Vertebral Torácica e Lombar. Cochrane Database Syst Rev. 2013;05:CD009073.
}

\section{Analysis of the Cochrane Review: Pedicle Screw Fixation for Traumatic Fractures of the Thoracic and Lumbar Spine. Cochrane Database Syst Rev. 2013;05:CD009073.}

\author{
Daniela LINHARES $\square^{1,2}$, Nuno NEVES ${ }^{1,3}$, Manuel Ribeiro da SILVA ${ }^{1,3}$, João Almeida FONSECA ${ }^{2,4}$
}

Acta Med Port 2016 May;29(5):297-300 - http://dx.doi.org/10.20344/amp.7687

\section{RESUMO}

As fraturas da coluna vertebral torácica e lombar são causa comum de cirurgia da coluna vertebral. A fixação com parafusos pediculares é geralmente escolhida para a sua estabilização, com recurso a instrumentações curtas, longas ou monosegmentares com ou sem enxerto ósseo. O objetivo desta revisão é avaliar o efeito da fixação transpedicular nas fraturas vertebrais torácicas e lombares traumáticas. Foi realizada uma pesquisa sistemática de estudos controlados aleatorizados ou quasi-aleatorizados que comparassem diferentes técnicas de tratamento cirúrgico destas fraturas, seguida de um processo de seleção, extração de dados e avaliação de viéses por três autores independentes. Oito estudos foram incluídos, num total de cinco comparações entre diferentes fixações com parafusos pediculares. Não se encontraram diferenças significativas quanto à melhoria da função e qualidade de vida, dos défices neurológicos ou da mobilidade. Apenas a instrumentação com parafusos pediculares no nível da fratura mostrou melhoria significativa da dor quando comparada com instrumentação posterior sem parafuso no nível. Várias técnicas resultaram em melhoria significativa dos parâmetros imagiológicos, sem melhoria clínica correspondente. De modo significativo tempos cirúrgicos mais curtos associaramse a menores perdas sanguíneas. O uso de enxerto ósseo causou um aumento significativo das complicações pós-operatórias, nomeadamente dor no local dador. Assim, os resultados mostram que melhorias imagiológicas significativas não se associam a impactos clínicos correspondentes, e que apenas o uso de fixação transpedicular no nível da fratura se associa a melhoria clínica evidente da dor. Adicionalmente, a necessidade de enxerto ósseo é posta em causa, pela ausência de melhoria clínico-imagiológica, com aumento das complicações. Contudo, o número de estudos controlados disponíveis acerca deste tópico é reduzido.

Palavras-chave: Fixação de Fractura; Fracturas da Coluna Vertebral; Parafusos Ósseos; Revisão Sistemática; Vértebras Lombares/ lesões; Vértebras Torácicas/lesões.

\section{ABSTRACT}

Traumatic fractures of the thoracic and lumbar spine are common causes of spine surgery. Pedicle screw fixation is usually chosen, using monosegmentar, short or long segment instrumentations, with or without bone graft. This review aims to evaluate the effect of transpedicular fixation in traumatic fractures of the thoracic and lumbar spine. A systematic search on controlled, randomized or quasirandomized trials comparing different methods of surgical treatment of this fractures was performed, followed by a process of article selection, data extraction and bias assessment by 3 independent authors. Eight articles were included in a total of 5 comparisons, between different transpedicular fixation techniques. No significant differences on function or quality of life, neurologic status or limitation of motion were found. Only instrumentation with fracture level screw incorporation showed significant decrease of pain when compared with instrumentation alone. Several techniques resulted in significant improvements of different radiological parameters Significantly, surgeries with smaller duration were associated with lesser blood loss. Bone graft use caused a significant raise in postoperative complications, namely donor site pain. So, this paper showed that significative improvements in radiological parameters do not associate with correspondent clinical benefits, and only instrumentation with level screw incorporation is associated with a clear benefit on pain. Moreover, the need for bone graft is questioned, since it leads to no clinic-radiological improvement with a raise of complications. However, a small number of controlled studies is available on this topic.

Keywords: Bone Screws; Fracture Fixation; Lumbar Vertebrae/injuries; Randomized Controlled Trials as Topic; Spinal Fractures/ surgery; Thoracic Vertebrae/injuries.

\section{INTRODUÇÃO}

As fracturas vertebrais torácicas e lombares são as lesões mais comuns da coluna vertebral (CV), podendo associar-se a importantes complicações quer imediatas, quer a longo prazo. Quando presentes critérios clínicos e/ ou radiológicos que assim o indiquem, a estabilização ci- rúrgica destas fracturas é efetuada. Existem diferentes técnicas cirúrgicas mas, a maioria das vezes, é realizada a fixação posterior com parafusos pediculares, que permite a estabilização das três colunas de Denis e um melhor alinhamento da coluna vertebral. A fixação transpedicular

1. Serviço de Ortopedia. Centro Hospitalar São João. Porto. Portugal.

2. Departamento de Ciências de Informação e Decisão em Saúde. Centro de Investigação em Tecnologias e Serviços de Saúde. Faculdade de Medicina da Universidade do Porto. Porto. Portugal.

3. Serviço de Ortopedia. Hospital CUF. Porto. Portugal.

4. Unidade de Imunoalergologia. Hospital CUF. Porto. Portugal.

$\square$ Autor correspondente: Daniela Linhares. daniela_linhares@sapo.pt

Recebido: 30 de março de 2016 - Aceite: 22 de abril de 2016 | Copyright (C) Ordem dos Médicos 2016 
posterior pode ser realizada recorrendo a instrumentação longa, curta ou monosegmentar com parafusos pediculares, e pode ainda associar-se à colocação de enxerto ósseo por via transpedicular. Contudo, nenhuma destas técnicas é livre de complicações e nenhum consenso foi ainda atingido no que respeita quer à escolha da técnica cirúrgica per se, quer à escolha do método de instrumentação transpedicular.

Assim, os autores efetuaram uma revisão sistemática de estudos que abordaram a seguinte questão clínica: qual o efeito da fixação transpedicular nas fraturas traumáticas das vértebras lombares e torácicas?

\section{MATERIAL E MÉTODOS}

Nesta revisão foram incluídos estudos controlados, randomizados (RCT) e quasi-randomizados, que comparam diferentes técnicas de fixação com parafusos pediculares, em doentes com fracturas traumáticas da CV lombar e torácica, com pelo menos $80 \%$ dos indivíduos tratados até três semanas após a lesão. Foram comparados:

1) Fixação com parafusos pediculares versus outro tipo de tratamento cirúrgico sem parafusos pediculares;

2) Diferentes métodos de fixação transpedicular: ins- trumentação curta, instrumentação longa, instrumentação curta com enxerto transpedicular; instrumentação curta com parafuso pedicular na vértebra fraturada; e instrumentação transpedicular monosegmentar. Como outcomes primários foram definidos: a) qualidade de vida e função auto-reportados; b) melhoria ou deterioração neurológica e c) dor. Como outcomes secundários foram considerados: as complicações pós-operatórias; a avaliação imagiológica; as perdas de sangue intra-operatórias e necessidade de transfusão; a duração da cirurgia; o impacto no emprego; a duração do internamento.

Foram efetuadas pesquisas nas bases de dados: Cochrane Bone, Joint and Muscle Trauma Group Specialised Register, Cochrane Central Register of Controlled Trials, MEDLINE, EMBASE e Chinese Biomedical Database, bem como outras pesquisas adicionais. A seleção dos artigos, a extração de dados e a avaliação dos riscos de viéses foi realizada independentemente por três autores e as discrepâncias foram resolvidas por discussão. Os autores foram contactados quando os dados eram insuficientes ou dúbios. Os resultados são apresentados como riscos relativos ou diferenças de médias, e IC 95\% para os outcomes dicotómicos ou contínuos, respetivamente.

Tabela 1 - Resumo dos resultados das comparações efetuadas

$\begin{array}{ccc}\text { Comparações } & \text { Função } \\ \text { (A vs. B) } & n & \begin{array}{c}\text { auto-reportada e } \\ \text { qualidade de vida }\end{array}\end{array}$

$\begin{array}{cc}\text { Status } \\ \text { Dor } & \text { neurológico } \\ \text { pós-operatório }\end{array}$

Complicações pós-operatórias
Correção compressão anterior
Fixação pedicular com parafusos vs.

outra cirurgia que não fixação pedicular com parafusos

Instrumentação curta vs.

instrumentação longa

Instrumentação curta com enxerto transpedicular

vs.

instrumentação curta

Instrumentação posterior com parafuso nível da fratura

vS.

instrumentação posterior

Instrumentação monosegmentar com parafuso pedicular vs.

instrumentação curta

Instrumentação posterior com fusão vs.

3

instrumentação posterior

\section{0}

2

Q

Q

NI/NA*

NA

$5,03 \%$

$(2,12 \text { a } 7,94)^{\$}$
2

1

1

Q

Q

NI/NA

NA

$-0,70$

$(-1,40 \text { a } 0,00)^{\$}$

Q

NA

(TVP; FI; Infeção)

$-3,86 \%$

$(-7,29 \text { a }-0,43)^{\$}$

Q
Q

NA

NA

Q

FI: $Q$

DSP:个

31,67

$(4,48 \text { a } 224,11)^{2}$

Q: sem diferenças estatisticamente significativas A vs. B; $\downarrow$ : valores de A significativamente inferiores vs. valores de $\mathrm{B}(p<0,05)$; $\uparrow$ : valores de A significativamente superiores $v s$. valores de *: heterogeneidade elevada; \#: os dados disponíveis não são claros; \$: diferença média (IC 95\%); \&: risco relativo (IC 95\%); vs.: versus; n: número de artigos que incluem a comparação; TVP: 


\section{RESULTADOS}

Foram encontrados 361 estudos dos quais oito tinham critérios para serem incluídos na análise final: cinco RCT e três estudos quasi-randomizados controlados, com um total de 448 participantes com idades médias entre 33 - 41 anos, e $64-83 \%$ de homens. Todos os estudos apresentaram critérios previamente definidos para indicação cirúrgi$\mathrm{ca}$, na presença de fratura da CV toracolombar.

Nenhum estudo realizou a comparação entre a fixação transpedicular com parafusos versus outro método cirúrgico que não fixação transpedicular com parafusos. Sete estudos apresentavam dois grupos de intervenção, e um estudo apresentava quatro grupos de intervenção, permitindo as comparações já descritas entre diferentes métodos de fixação transpedicular com parafusos.

Todos os estudos efetuaram avaliação da função e qualidade de vida auto-reportadas através da utilização de diferentes questionários; cinco estudos restringiram a inclusão a indivíduos sem lesões neurológicas; dois estudos efetuaram avaliação independente da dor e seis avaliaram-na como componente de outras escalas. Todos fazem referência à ocorrência de complicações pós-operatórias, aos resultados dos outcomes imagiológicos e peri-operató- rios. Nenhum estudo apresentou dados acerca do emprego. Seis estudos apresentam o tempo de internamento dos doentes estudados.

Na Tabela 1 são sumariados os resultados das análises dos diferentes outcomes relativamente às comparações atrás referidas.

\section{COMENTÁRIO}

Esta revisão mostra que as diferentes montagens que envolvem fixação com parafusos pediculares não diferem significativamente entre si, não permitindo recomendar inequivocamente uma técnica em particular.

Não foram encontrados estudos que comparem a fixação com parafusos pediculares com fixações que não recorram ao uso destes parafusos, sendo que a revisão sistemática apresentada se debruça apenas sobre as diferentes alternativas de fixação transpedicular. De forma semelhante, uma revisão sistemática sobre o tratamento cirúrgico deste tipo de fraturas, não limitada a RCTs, demonstrou que os parafusos pediculares são a forma mais consensual e frequente de tratamento destas lesões, com outras técnicas diferentes a serem descritas apenas esporadicamente, em situações específicas. ${ }^{1}$ Também uma outra revisão

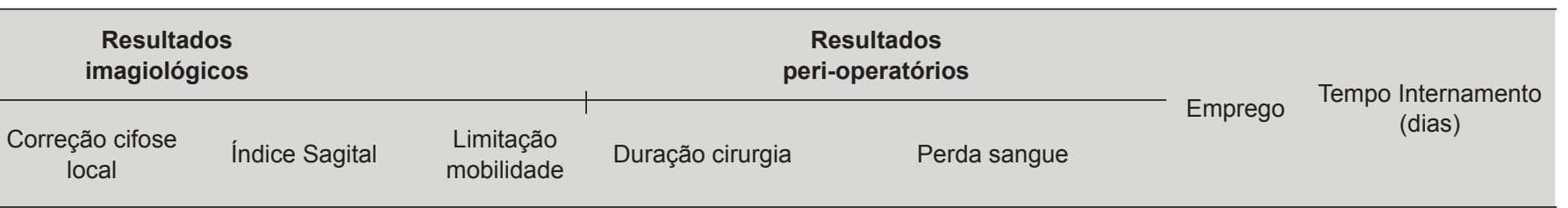

$\boldsymbol{\nu}$

$2,98^{\circ}$ $(0,96 \text { a } 5,01)^{\$}$

Q

个*

$-3.04^{\circ}$

$(-5,73 \text { a }-0,35)^{\$}$ $\theta$

$-$ $\theta$

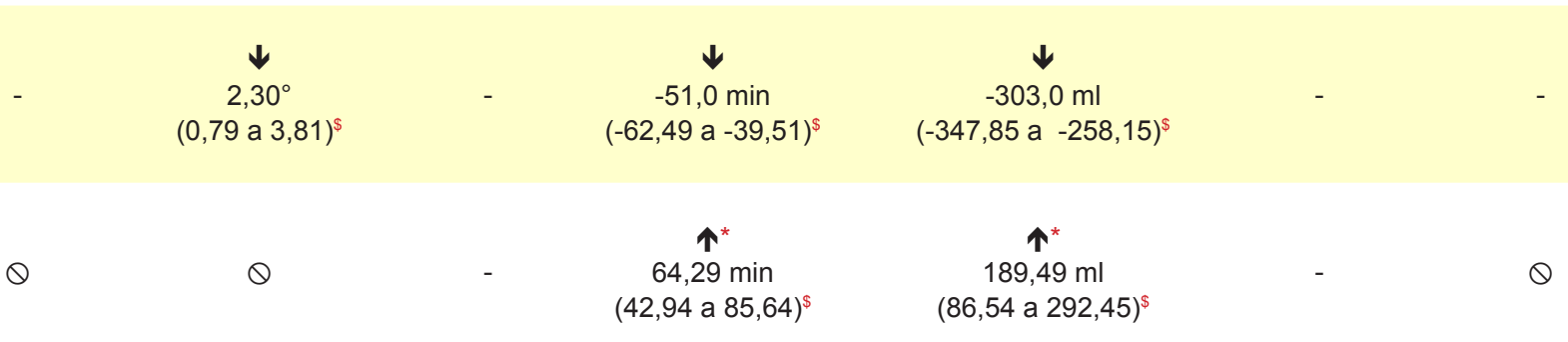

$-9,92 \min$

$(-19,31 \text { a }-0,54)^{\$}$ $\theta$

$-1,28$ dias

$(-2,16 \text { a }-0,40)^{s}$ 
sistemática recente sobre fraturas tipo burst concluiu que, embora não haja consenso acerca da técnica a ser usada, os estudos de maior nível de evidência mostram uma tendência para a escolha da fixação transpedicular. ${ }^{2}$

Face à evidência atual que favorece a utilização da fixação transpedicular é improvável que no futuro surjam estudos que permitam uma comparação adequada entre este tipo de fixação e técnicas previamente descritas.

A revisão apresentada mostra que os diferentes tipos de fixação transpedicular não diferem no que respeita ao resultado funcional e impacto na qualidade de vida, e apenas a instrumentação posterior com inclusão do nível fraturado obteve resultados mais benéficos em termos de redução da dor, quando comparada com a instrumentação posterior que não inclua o nível fraturado.

Os principais resultados desta revisão incidem sobre melhorias na correção imagiológica. Interessantemente, a maioria destes resultados imagiológicos não se parece traduzir em melhorias nos parâmetros de avaliação clínica, nomeadamente capacidade funcional, qualidade de vida ou dor.

Esta revisão confirma ainda que cirurgias com menores durações (instrumentações curtas ou monosegmentares) se associam, geralmente, a menores perdas sanguíneas.

Poucos RCTs estão disponíveis no que respeita ao re- sultado dos diferentes tipos de fixações em doentes com fraturas da coluna toracolombar. Salienta-se que esta revisão incluiu apenas oito estudos, e os resultados apresentados provêm de meta-análises com três ou menos artigos, com heterogeneidade elevada em várias comparações, sendo que em duas delas apenas um RCT está disponível.

\section{IMPLICAÇÕES PARA A PRÁTICA}

Os autores questionam a necessidade do uso de autoenxerto ósseo, dado que os resultados dos estudos não observaram uma melhoria clínica ou imagiológica da fixação nestes casos, havendo um aumento significativo da dor no local de colheita. Adicionalmente, verificou-se que algumas técnicas se associam a menores tempos cirúrgicos e menores perdas sanguíneas, o que pode ser importante em casos específicos de doentes com co-morbilidades, menos tolerantes a perdas sanguíneas e a longos tempos anestésicos.

Dado apenas estarem disponíveis oito estudos elegíveis de acordo com os critérios de análise, são necessários mais ensaios clínicos controlados, com critérios homogéneos, definidos por consensos internacionais, que comparem os resultados das diferentes técnicas de fixação em doentes com fraturas traumáticas da CV toracolombar.

\section{REFERÊNCIAS}

1. Verlaan JJ, Diekerhof CH, Buskens E, Tweel I, Verbout AJ, Dhert WJA, et al. Surgical treatment of traumatic fractures of the thoracic and lumbar spine. Spine. 2004;7:803-14.

2. Scheer JK, Bakhsheshian J, Fakurnejad S, Oh T, Dahdaleh NS,

Smith ZA. Evidence-based medicine of traumatic thoracolumbar burst fractures: a systematic review of operative management across 20 years. Global Spine J. 2015;5:73-82.

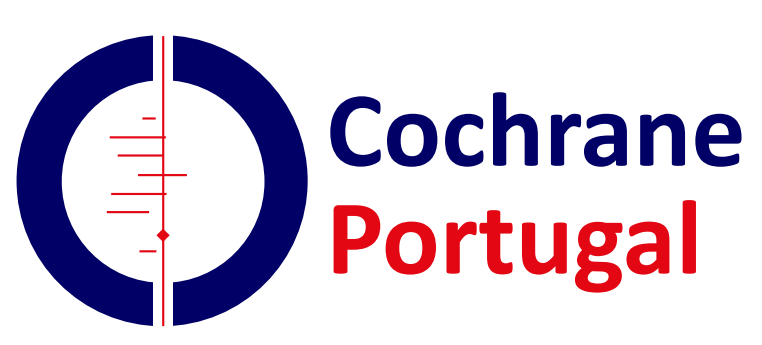

